Pacific Journal of Mathematic 


\title{
FUNDAMENTAL UNITS AND CYCLES IN THE PERIOD OF REAL QUADRATIC NUMBER FIELDS
}

\author{
LEON BERNSTEIN
}

\section{PART I}

0. Introduction. In this paper we introduce the concept of "Cycles in the Period" of the simple continued fraction expansion of a real quadratic irrational. This is expressed in the

Definition. Let $M, D, d$ be positive rational integers, $M$ sequare free, $M=D^{2}+d, d \leqq 2 D$. Let $k, a, s$ be nonnegative rational integers, $0 \leqq a \leqq k-1$; let $f=f(k, a, s ; d, D)$ be a polynomial with rational integral coefficients. For a fixed $s$, the finite sequence of polynomials

$$
\begin{aligned}
F) s)= & f(k, a, s ; d, D), f(k, a+1, s ; d, D), \cdots, \\
& f(k, a+k-1, s ; d, D)
\end{aligned}
$$

will be called "Cycle in the Period" of the simple continued fraction expansion of $\sqrt{ } \bar{M}$ if, for $s_{0} \geqq 1$, this expansion has the form

$$
\begin{aligned}
\sqrt{M}= & {\left[\overline{b_{0}, b_{1}, \cdots, F(0), \cdots, F\left(s_{0}-1\right), f\left(k, a, s_{0} ; d, D\right), \cdots},\right.} \\
& \overline{f\left(k, a+b, s_{0} ; d, D\right), \cdots, f\left(k, a, s_{0} ; d, D\right), F^{\prime}\left(s_{0}-1\right), \cdots}, \\
& \overline{\left.F^{\prime}(0), f(k, a-1,0 ; d, D), \cdots, b_{1}, 2 b_{0}\right]}
\end{aligned}
$$

$b \geqq 1 ; b \leqq k-1 ; k$ is the length of the cycle; $F^{\prime}(s)$ means that the order of the $f-s$ must be reversed.

In the first part of this paper, the main result is the construction of infinitely many classes of quadratic fields $Q(\sqrt{M})$, each containing infinitely many $M$ of a simple structure. Among the various classes thus constructed, there are a few in whose expansion of $\sqrt{M}$ cycles in the period surprisingly have the length $\leqq 12$. Functions $f(k, a, s ; d, D)$, $f(k, a+1, s ; d, D), \cdots$ are of course stated explicitly; hence we are able to construct numbers $\sqrt{\bar{M}}$ such that the primitive period of their expansion has any given length $m$ which is a function of the parameter $k$.

Expansions of $\sqrt{M}$ which have the structure of cycles in the period were generally not known up to now. In a recent paper $Y$. Yamamoto [6] has given a few numerical examples of expantions of 
real quadratic irrationals in which cycles of length two appear. The present paper, which generalizes this concept, was, however, developed independently of Yamoto's beautiful discovery.

It is an old dream of mathematicians to find infinite classes of real quadratic number fields $Q(\sqrt{M})$ for which the continued fraction expansion can be stated explicitly as a function of $M$. Very little knowledge in this direction was available up to now. The bit of it that was there is based on a theorem by Th. Muir [4], and in his "encyclopedia" of continued fractions O. Perron [5] has given a few demonstrations of Muire's theorem which the author does not believe to be of great practical significance. The most that can be achieved with Muir's theorem, is the explicitly stated expansion of certain classes of $\sqrt{M}$ with a primitive period up to length six. In a recent paper [2] the author has given the following infinite classes of $\sqrt{M}$ whose continued fraction expansion has a primitive period of lengths 10, 12 and 8 respectively; these are

$$
\begin{aligned}
& \sqrt{D^{2}+4 d} \\
& =\left[D, \overline{(2 d)^{-1}(D-d), 1,1,2^{-1}(D-1), 2 d^{-1} D, 2^{-1}(D-1)}\right. \\
& \quad \overline{\left.1,1,(2 d)^{-1}(D-d), 2 D\right]} \\
& \quad d \mid D ; d>1, D \text { odd } ; D>d
\end{aligned}
$$

$$
\sqrt{D^{2}-4 d}
$$

$$
\begin{aligned}
= & {\left[D-1, \overline{1,(2 d)^{-1}(D-3 d), 2,2^{-1}(D-3), 1,2 d^{-1}(D-d)},\right.} \\
& \overline{\left.1,2^{-1}(D-3), 2,(2 d)^{-1}(D-3 d), 1,2 b_{0}\right]} \\
& d \mid D ; d>1, D \text { odd; } b_{0}=D-1 ; D^{2}-4 d \text { squarefree } .
\end{aligned}
$$

$$
\begin{aligned}
& \sqrt{9 d^{2}-4 d} \\
& =\left[3 d-1, \overline{\left.3,3(d-1) 2^{-1}, 1,4,1,3(d-1) 2^{-1}, 3,2(3 d-1)\right]}\right. \\
& \quad d>1, d \text { odd } .
\end{aligned}
$$

This is a special case of of (0.9)

For $d=1$, the primitive period in the above expansions has length at most half of the original one, as the reader verify easily. Then author constructed these expansions in order to find the wellknown fundamental unit of G. Degert [3] in the corresponding quadratic field by an approach different from Degert's.

We shall recall the basic rules of expanding $\sqrt{M}$ for further references:

$$
\begin{aligned}
\sqrt{M} & =w=x_{0}=\left(w+P_{0}\right) Q_{0}^{-1}=b_{0}+x^{-1} ; P_{0}=0 ; Q_{0}=1 ; b_{0} \\
& =[w] .
\end{aligned}
$$




$$
\begin{aligned}
x_{k} & =\left(w+P_{k}\right) Q_{k}^{-1}=b_{k}+x_{k+1}^{-1} ; P_{k}=b_{k-1} Q_{k-1}-P_{k-1} ; Q_{k-1} Q_{k} \\
& =w^{2}-P_{k}^{2} ; b_{k}=\left[x_{k}\right] ;(k=1,2, \cdots) .
\end{aligned}
$$

From $P_{k}+P_{k-1}=b_{k-1} Q_{k-1}, w^{2}-P_{k}^{2}=Q_{k-1} Q_{k}$, we derive the formula

$$
\begin{array}{r}
\left(w+P_{k-1}\right)\left(w+P_{k}\right)=Q_{k-1}\left(Q_{k}+b_{k-1}\left(w+P_{k}\right)\right) \\
\quad(k=1,2, \cdots) .
\end{array}
$$

With $m$ denoting the length of the primitive period of $\sqrt{M}$ as a periodic continued fraction expansion, the following rules hold:

$$
\text { Let } m=2 k \text {; then } P_{k}=P_{k+1} \text {, and vice versa; also } b_{k-i}=
$$$$
b_{k+i} ;(i=1, \cdots, k) ; b_{2 k}=2 b_{0}^{k} \text {. }
$$

Let $m=2 k+1$; then $Q_{k}=Q_{k+1}$, and vice versa; also $b_{k}=b_{k+1} ; b_{k-i}=b_{k+i+1}(i=1, \cdots, k-1) ; b_{2 k+1}=2 b_{0}$. If $m=2 k$, then $P_{k-i}=P_{k+i+1}(i=0, \cdots, k-1) ; Q_{k-i}=$ $Q_{k+i}(i=1, \cdots, k)$.

If $m=2 k+1$, then $P_{k-i}=P_{k+i+2}(i=0, \cdots, k-1)$; $Q_{k-i}=Q_{k+i+1}(i=0, \cdots, k)$.

$Q_{v}>2(v=1,2 \cdots)$. Only if $m=2 k, Q_{k}=2$ is possible (then $P_{k}=P_{k+1}$ ).

In Part II (later in this volume) the fundamental unit of $Q(\sqrt{M}), M$ square free, is stated explicitly, being calculated from the periodic expansion of $\sqrt{M}$.

The explicit representation of this expansion is therefore a primary issue. Units of algebraic number fields of any degree have been recently investigated by $\mathrm{H}$. Zassenhaus [7]. This author also thinks that the calculation of units from the periodic expansion of a basis of the field, generally by Jacobi-Perron algorithm, is a most suitable tool.

In the following chapters $w^{2}$ is squarefree.

1. Expansion of $w=\sqrt{\left[(2 a+1)^{k}+a\right]^{2}+2 a+1} ; a, k \geqq 1$. The formula holds

$$
\begin{gathered}
w^{2}=A^{2 k}+2 a A^{k}+(a+1)^{2}=\left(A^{k}+a\right)^{2}+A ; A=2 a+1 . \\
{[w]=A^{k}+a ; w=A^{k}+a+r ; 0<r<1 .}
\end{gathered}
$$

The reader will easily verify the following expansion for $k \geqq 2$.

$$
w=A^{k}+a+\frac{1}{x_{1}} ; P_{1}=A^{k}+a ; Q_{1}=A ;
$$




$$
\frac{w+A^{k}+a}{A}=2 A^{k-1}+\frac{1}{x_{2}} ; P_{2}=A^{k}-a ; Q_{2}=4 a A^{k-1}+1
$$

$$
\begin{gathered}
\frac{w+A^{k}-a}{4 a A^{k-1}+1}=1+\frac{1}{x_{3}} ; P_{3}=(A-2) A^{k-1}+a+1 ; Q_{3}=2 A^{k-1} \\
\frac{w+(A-2) A^{k-1}+a+1}{2 A^{k-1}}=A-1+\frac{1}{x_{4}} ; P_{4}=A^{k}-(a+1) \\
Q_{4}=A^{2} \\
\frac{w+A^{k}-(a+1)}{A^{2}}=2 A^{k-2}-1+\frac{1}{x_{5}}
\end{gathered}
$$

We now prove the formulas

$$
\left\{\begin{aligned}
& \text { ( i ) } P_{3 s-1}= A^{k}-\left[A^{s}-(a+1)\right] ; Q_{3 s-1}=2\left(A^{s}-1\right) A^{k-s} \\
&-\left[A^{s}-2(a-1)\right] ; b_{3 s-1}=1 \\
& \text { (ii) } \quad P_{3 s}=\left(A^{s}-2\right) A^{k-s}+(a+1) ; Q_{3 s}=2 A^{k-s} ; b_{3 s}=A^{s}-1 \\
& \text { (iii) } \quad P_{3 s+1}=A^{k}-(a+1) ; Q_{3 s+1}=A^{s+1} ; b_{3 s+1}=2 A^{k-(s+1)}-1 .
\end{aligned}\right.
$$

Proof by idduction. Formulas (1.8) are correct for $s=1$, as can be easily verified from (1.3) to (1.7). We prove: (1.8) is true for $s$ being replaced by $s+1$. We obtain from (1.8), (iii), presuming $1 \leqq s+1 \leqq k$,

$$
\left\{\begin{array}{c}
\frac{w+A^{k}-(a+1)}{A^{s+1}}=2 A^{k-(s+1)}-1+\frac{1}{x_{3 s+2}} ; P_{3 s+2} \\
=A^{k}-\left[A^{s+1}-(a+1)\right] \\
Q_{3 s+2}=2\left(A^{s+1}-1\right) A^{k-(s+1)}-\left[A^{s+1}-2(a+1)\right] .
\end{array}\right.
$$

Since

$$
\begin{aligned}
1< & 1+\frac{2 A^{k-(8+1)}-1+r}{Q_{3 s+2}}=x_{3 s+2}<1+\frac{2 A^{k-2}}{2 A^{k}-2 A^{k-1}-A^{k}} \\
& =1+\frac{2}{(2 a-1) A}<2
\end{aligned}
$$

we obtain

$$
\left\{\begin{array}{l}
\frac{w+A^{k}-A^{s+1}+(a+1)}{2 A^{k}-2 A^{k-(s+1)}-A^{s+1}+2(a+1)}=1+\frac{1}{x_{3 s+3}} \\
P_{3 s+3}=\left(A^{s+1}-2\right) A^{k-(s+1)}+(a+1) ; Q_{3 s+3}=2 A^{k-(s+1)} .
\end{array}\right.
$$

Since, for $k \geqq 2$,

$$
A^{s+1}-1<A^{s+1}-1+\frac{A+r}{2 A^{k-(s+1)}}=x_{3 s+3}<A^{s+1}-1+\frac{2 A}{2 A^{k-(s+1)}} \leqq A^{s+1},
$$


we obtain

$$
\left\{\begin{array}{l}
\frac{w+A^{k}-2 A^{k-(s+1)}+a+1}{2 A^{k-(s+1)}}=A^{s+1}-1+\frac{1}{x_{3 s+4}} \\
P_{3 s+4}=A^{k}-(a+1) ; Q_{3 s+4}=A^{s+2} .
\end{array}\right.
$$

Since

$2 A^{k-(s+2)}-1<2 A^{k-(s+2)}-1+\frac{A^{s+2}-1+r}{A^{s+2}}=x_{3 s+4}<2 A^{k-(s+2)}-1+1$, we have

$$
b_{3 s+4}=2 A^{k-(s+2)}-1 .
$$

With formulas (1.9)-(1.12) formulas (1.8), with $s$ replaced by $s+1$ are verified. Since, as can easily be verified from (1.8), no $P_{v}$ equals $P_{v+1}$, and no $Q_{v}$ equals $Q_{v+1}$ in the cycle $\left\{P_{3 s-1}, P_{3 s}, P_{3 s+1}\right\}$ and $\left\{Q_{3 s-1}, Q_{3 s}, Q_{3 s+1}\right\}$. We look for the the possibility that some $Q_{v}=2$. This happens for

$$
Q_{3 k}=2 \text {. }
$$

We now obtain from (1.8), (ii), and from (1.2)

$$
\left\{\begin{array}{l}
\frac{w+A^{k}+a-1}{2}=\frac{2 A^{k}+2 a-r^{\prime}}{2}=A^{k}+a-1+\frac{1}{x_{3 k+1}} \\
P_{3 k+1}=A^{k}+a-1=b_{0}-1=P_{3 k} .
\end{array}\right.
$$

The length of the primitive period of the expansion of $w$ thus equals $1+6(k-1)+3+2=6 k$, and we can state.

THEOREM 1. Let $a, k$ be natural numbers, $a \geqq 1, k \geqq 2, w^{2}=$ $\left(A^{k}+a\right)^{2}+A, A=2 a+1, w^{2}$ squarefree. The expansion of $w$ as $a$ periodic continued fraction has a primitive period of length $6 \mathrm{k}$ and the form

$$
\left\{\begin{array}{l}
w=\overline{\left[b_{0}, b_{1}, \cdots, b_{3 s-1}, b_{3 s}, b_{3 s+1}, \cdots, b_{3 k-1}, b_{3 k}, b_{3 k-1}, \cdots, b_{1}, 2 b_{0}\right]} \\
b_{0}=A^{k}+a ; b_{1}=2 A^{k-1} ; b_{3 k-1}=1 ; b_{3 k}=b_{0}-1 \\
b_{3 s-1}=1 ; b_{3 s}=A^{s}-1 ; b_{3 s+1}=2 A^{k-s-1}-1 ; s=1, \cdots, k-1 .
\end{array}\right.
$$

For $k=1$, we obtain by a special calculation that

$$
\sqrt{9 a^{2}+8 a+2}=[3 a+\overline{2,1,3 a, 1,2,6 a+2}] .
$$

2. Expansion of $w=\sqrt{\left(A^{k}-a\right)^{2}+A} ; A=2 a+1 ; a, k \geqq 1$. The formulas hold 


$$
\begin{gathered}
w^{2}=A^{2 k}-2 a A^{k}+(a+1)^{2} . \\
{[w]=A^{k}-a ; w=A^{k}-a+r, 0<r<1 .}
\end{gathered}
$$

The reader will easily verify the following

Theorem 2. Let $a, k$ be natural numbers, $a \geqq 1, k \geqq 2, w^{2}=$ $\left(A^{k}-a\right)^{2}+A, A=2 a+1, w^{2}$ squarefree. The expansion of $w$ as a periodic continued fraction has a primitive period of length $6 k-2$ and the form

$$
\begin{aligned}
& w=\left[b_{0}, \cdots, b_{3 s-2}, b_{3 s-1}, b_{3 s}, \cdots, b_{3 k-2}, b_{3 k-1}, b_{3 k-2}, \cdots, b_{1}, 2 b_{0}\right] \\
& \left\{\begin{array}{l}
b_{0}=A^{k}-a ; b_{3 k-1}=b_{0}-1 ; b_{3 k-2}=1 \\
b_{3 s-2}=2 A^{k-s}-1 ; b_{3 s-1}=A^{s}-1 ; b_{3 s}=1 ; s=1, \cdots, k-1
\end{array}\right.
\end{aligned}
$$

For $k=1$, we obtain the expansion

$$
\sqrt{a^{2}+4 a+2}=[a+1, \overline{1, a, 1,2(a+1)}] .
$$

(2.6) is obtained from $\sqrt{a^{2}+4 a+2}=\sqrt{(a+2)^{2}-2}$, for which the author has found (2.6) in [1].

3. Expansion of $w=\sqrt{\left(A^{k}+a+1\right)^{2}-A} ; A=2 a+1 ; a, k \geqq 1$. The formulas hold

$$
\begin{gathered}
w^{2}=A^{2 k}+2(a+1) A^{k}+a^{2} ; \\
{[w]=A^{k}+a ; w=A^{k}+a+r ; 0<r<1 .}
\end{gathered}
$$

The reader will easily verify the following

THEOREM 3. Let $a, k$ be natural numbers, $a \geqq 1, k \geqq 2 ; w^{2}=$ $\left(A^{k}+a+1\right)^{2}-A ; A=2 \alpha+1, w^{2}$ squarefree. The expansion of $w$ as a periodic continued fraction has a primitive period of length $4 k+2$ and the form

$$
\left\{\begin{array}{l}
w=\left[b_{0}, \overline{\left.\cdots, b_{2 s-1}, b_{2 s}, \cdots, b_{2 k+1}, b_{2 k}, \cdots, b_{1}, 2 b_{0}\right]}\right. \\
b_{0}=b_{2 k+1}=A^{k}+a ; b_{2 s-1}=A^{s-1} ; b_{2 s}=2 A^{k-s} \\
s=1, \cdots, k .
\end{array}\right.
$$

For $k=1$ we obtain the expansion

$$
\sqrt{9 a^{2}+10 a+3}=[3 a+\overline{1,1,2,3 a+1,2,1,6 a+2}] .
$$

4. Expansion of $w=\sqrt{\left[A^{k}-(a+1)\right]^{2}-A} ; A=2 a+1, a, k \geqq 2$. The formulas hold

$$
w^{2}=A^{2 k}-2(a+1) A^{k}+a^{2}
$$




$$
\begin{aligned}
{[w] } & =A^{k}-(a+2) ; \\
w & =A^{k}-a-2+r ; 0<r<1 .
\end{aligned}
$$

The reader will easily verify the following:

TheOREM 4. Let $a, k \geqq 1$ be natural numbers, $w^{2}=\left[A^{k}-(a+1)\right]^{2}-$ $A, A=2 a+1, w^{2}$ squarefree. The expansion of $w$, as a periodic continued fraction, has a primitive period of length $4(2 k-1)$ and the form

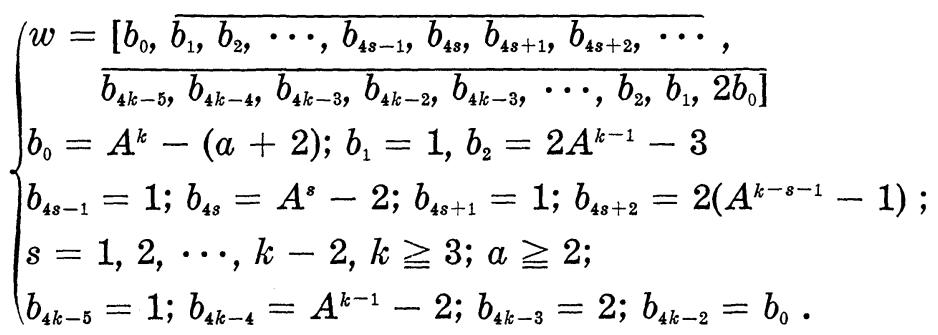

For $k=2, a \geqq 1$ the expansion holds

$$
\begin{aligned}
& \sqrt{\left.\left(4 a^{2}+3 a\right)^{2}-2 a+1\right)} \\
& \quad=\left[4 a^{2}+3 a-1, \overline{1,4 a-1,1,2 a-1,2,4 a^{2}+3 a-1,2}\right. \\
& \quad \frac{\left.2 a-1,1,4 a-1,1,8 a^{3}+6 a-2\right]}{} .
\end{aligned}
$$

For $k=1, a \geqq 4$, the expansion holds

$$
\sqrt{a^{2}-2 a-1}=[a-2, \overline{1, a-3,1,2(a-2)}] .
$$

5. Expansion of $w=\sqrt{\left[A^{k}+(A-1)\right]^{2}+4 A ;} A=2^{d} b, b$ odd $\geqq$ $1 ; d \geqq 1 ; k \geqq 2$. The formulas hold ( $d$ and $b$ not both equal 1 )

$$
\begin{aligned}
w^{2} & =A^{2 k}+2(A-1) A^{k}+(A+1)^{2} ;[w] \\
& =A^{k}+A-1 ; w=[w]+r, 0<r<1 .
\end{aligned}
$$

The reader will easily verify the following:

TheOREM 5. Let $A=2^{d} b, b$ odd $\geqq 1, d \geqq 2, k \geqq 2$ be natural numbers, $w^{2}=\left[A^{k}+(A-1)\right]^{2}+4 A$ squarefree. If $k \geqq 3$ is odd, then the expansion of $w$, as a periodic continued fraction has a primitive period of length $5 k-6$. The expansion has the form

$$
\left\{\begin{array}{c}
w=\frac{\left[b_{0}, \overline{b_{1}, \cdots, b_{5 s-3}, b_{5 s-2}, b_{5 s-1}, b_{5 s}, b_{5 s+1}, \cdots},\right.}{\left.b_{1 / 2(5 k-11)}, b_{1 / 2(5 k-9)}, b_{1 / 2(5 k-7)}, b_{1 / 2(5 k-7)}, b_{1 / 2(5 k-9)}, \cdots, b_{1}, 2 b_{0}\right]} \\
\text { (i) } b_{0}=A^{k}+A-1 ; b_{1}=2^{d-1} b A^{k-2} \\
\text { (ii) } b_{5 s-3}=2 ; b_{5 s-2}=2^{d-1} b A^{s-1}-1 ; b_{5 s-1}=b_{5 s}=1 ; b_{5 s+1} \\
=2^{d-1} b A^{k-s-2}-1 .
\end{array}\right.
$$




$$
\text { (iii) }\left\{\begin{array}{l}
s=1,2, \cdots, \frac{1}{2}(k-3), k \geqq 5 . \\
b_{1 / 2(5 k-11)}=2, \\
b_{1 / 2(5 k-9)}=2^{d-1} b A^{1 / 2(k-3)}-1, \\
b_{1 / 2(5 k-7)}=1 .
\end{array}\right.
$$

For $k=3$, the expansion has the form

$$
\left\{\begin{array}{l}
\sqrt{\left[A^{3}+(A-1)\right]^{2}+4 A} \\
\quad=\left[A^{3}+a-1, \overline{2^{d-1} b A, 2,2^{d-1} b-1,1,1,2^{d-1} b}\right. \\
\left.\quad-1,2,2^{d-1} b A, 2\left(A^{3}+A-1\right)\right]
\end{array}\right.
$$

If $k \geqq 2$ is even, the expansion of $w$, as a periodic continued fraction has a primitive period of length $5 k-6$. The expansion has the form

$$
\left\{\begin{array}{c}
w=\frac{\left[b_{0}, \overline{b_{1}, \cdots, b_{5 s-3}, b_{5 s-2}, b_{5 s-1}, b_{5 s}, b_{5 s+1}, \cdots, 2, \cdots,}\right.}{\left.b_{5 s+1}, b_{5 s}, b_{5 s-1}, b_{5 s-2}, b_{5 s-3}, \cdots, b_{1}, 2 b_{0}\right]} \\
s=1,2, \cdots, \frac{1}{2}(k-2) ; k \geqq 4 . \\
b_{0}, b_{1}, b_{5 s-3}, b_{5 s-2}, b_{5 s-1}, b_{5 s}, b_{5 s+1} \text { as in (5.17), (i), (ii) } .
\end{array}\right.
$$

For $k=2$, the expansion has the form

$$
\left\{\begin{array}{l}
\sqrt{\left[A^{2}+(A-1)\right]^{2}+4 A} \\
\left.\quad=\left[A^{2}+A-1, \overline{2^{d-1} b, 2,2^{d-1} b, 2\left(A^{2}+A-1\right.}\right)\right] .
\end{array}\right.
$$

Example 1. For $d=2, b=1, A=4$, we obtain from (5.18)

$$
\sqrt{4505}=[67, \overline{8,2,1,1,1,1,2,8,134}] \text {. }
$$

Example 2. For $d=2, b=1, A=4$, we obtain from (5.20)

$$
\sqrt{377}=[19, \overline{2,2,2,38}] \text {. }
$$

The reader should note that for $k=1$, we obtain the known expansion $\sqrt{4 A^{2}+1}=[2 A, \overline{4 A}]$.

As is known in the case of an odd period of length $2 r+1$, the formulas hold

$$
w^{2}=P_{r+1}^{2}+Q_{r+1}^{2} .
$$

In our case $5 k-6=2 r+1$, hence

$$
r+1=\frac{5}{2}(k-1),
$$


and so we obtain

$$
Q_{5 / 2(k-1)}^{2}+P_{5 / 2(k-1)}^{2}=\left[A^{k}+(A-1)\right]^{2}+4 A .
$$

We obtain from (5.8), (iv), for $s=1 / 2(k-1)$,

$$
\begin{aligned}
& Q_{5 / 2(k-1)}=\left(A^{1 / 2(k-1)}+1\right) A^{k-1 / 2(k-1)}-\left[A^{1 / 2(k-1)+1}+1(A+1)\right], \\
& P_{5 / 2(k-1)}=A^{k-(k-1) / 2}+A^{(k-1) / 2+1}, \\
& P_{5 / 2(k-1)}=2 A^{(k+1) / 2} ; Q_{5 / 2(k-1)}=A^{k}-(A+1) . \\
& \text { Indeed: } \\
& \begin{aligned}
P_{5 / 2(k-1)}^{2}+Q_{5 / 2(k-1)}^{2} & =4 A^{k+1}+A^{2 k}-2(A+1) A^{k}+(A+1)^{2} \\
& =A^{2 k}+2(A-1) A^{k}+(A+1)^{2}=w^{2},
\end{aligned}
\end{aligned}
$$

by $(5.1)$.

6. Expansion of $w=\sqrt{\left[A^{k}-(A-1)\right]^{2}+4 A}, A=2^{d} b, d \geqq 1, b \geqq$ 1 odd, $k \geqq 2$. The formulas hold ( $d$ and $b$ not both equal 1 )

$$
\begin{aligned}
w^{2} & =A^{2 k}-2(A-1) A^{k}+(A+1)^{2} ;[w]=A^{k}-(A-1) ; \\
w & =[w]+r, 0<r<1 .
\end{aligned}
$$

The reader will easily verify the following

THEOREM 6. Let $A=2^{d} b, b$ odd $\geqq 1, d \geqq 2$, be natural numbers, $w^{2}=\left[A^{k}-(A-1)\right]^{2}+4 A$ squarefree. If $k \geqq 3$ is odd, then the expansion of $w$ as a periodic continued fraction has a primitive period of length $5(k-1)$. The expansion has the form

$$
\left\{\begin{array}{l}
w=\frac{\left[b_{0}, \cdots, \overline{b_{5 s+1}, b_{5 s+2}, b_{5 s+3}, b_{5 s+4}, b_{5 s+5}, \cdots, b_{1 / 2(5 k-13)}, b_{1 / 2(5 k-11)}}\right.}{\left.b_{1 / 2(5 k-9)}, b_{1 / 2(5 k-7)}, b_{1 / 2(5 k-5)}, b_{1 / 2(5 k-7)}, b_{1 / 2(5 k-9)}, \cdots, 2 b_{0}\right]} \\
b_{5 s+1}=2^{d-1} b A^{k-s-2}-1 ; \\
b_{5 s+2}=1 ; \\
b_{5 s+3}=1 ; \\
b_{5 s+4}=2^{d-1} b A^{s}-1 ; \\
b_{5 s+5}=2 ; s=0,1, \cdots, \frac{1}{2}(k-5) ; \\
b_{1 / 2(5 k-13)}=2^{d-1} b A^{1 / 2(k-1)}-1 ; b_{1 / 2(5 k-11)}=b_{2(5 k-9)}=1 ; \\
b_{1 / 2(5 k-7)}=2^{d-1} b A^{1 / 2(k-3)}-1 ; b_{1 / 2(5 k-5)}=2 . \\
b_{0}=A^{k}-(A-1) .
\end{array}\right.
$$

For $k-3, w$ has the expansion

$$
\left\{\begin{array}{l}
\sqrt{\left[A^{3}-(A-1)\right]^{2}+4 A} \\
\quad=\left[A^{3}-A+1, \overline{2^{d-1} b A-1,1,1,2^{d-1} b-1,2,2^{d-1} b}\right. \\
\quad \frac{\left.-1,1,1,2^{d-1} b A-1,2\left(A^{3}-A+1\right)\right] .}{}
\end{array}\right.
$$


If $k \geqq 4$ is even then the expansion of $w$ as a periodic continued fraction has a primitive period of length $5 k-5$. The expansion has the form

$$
\left\{\begin{array}{l}
w=\left[b_{0}, \overline{\cdots b_{5 s+1}, b_{5 s+2}, b_{5 s+3}, b_{5 s+4}, b_{5 s+5}, \cdots,}\right. \\
\quad \frac{\left.b_{1 / 2(5 k-8)}, 1,1, b_{1 / 2(5 k-8)}, \cdots, 2 b_{0}\right]}{b_{0}=} A^{k}-(A-1) ; \\
b_{5 s+1}, b_{5 s+2}, b_{5 s+3}, b_{5 s+4}, b_{5 s+5} \text { as in }(6.12) ; s=0,1, \cdots, \frac{1}{2}(k-4), \\
b_{1 / 2(5 k-8)}=2^{d-1} b A^{1 / 2(k-2)}-1 .
\end{array}\right.
$$

For $k=2$, the expansion of $w$ is

$$
\left\{\begin{array}{l}
\sqrt{\left[A^{2}-(A-1)\right]^{2}+4 A} \\
\quad=\left[A^{2}-A+1, \overline{\left.2^{d-1} b-1,1,1,2^{d-1} b-1,2\left(A^{2}-A+1\right)\right]} .\right.
\end{array}\right.
$$

By formula (5.21) we obtain here

$$
\left\{\begin{array}{c}
P_{5 s+3}^{2}+Q_{5 s+3}^{2}=w^{2}, s=\frac{1}{2}(k-2) ; P_{1 / 2(5 k-4)}=2 A^{1 / 2 k} ; \\
A_{1 / 2(5 k-4)}=A^{k}-(A+1) .
\end{array}\right.
$$

Indeed:

$\left(2 A^{2 k}\right)^{2}+\left[A^{k}-(A+1)\right]^{2}=A^{2 k}-2(A-1) A^{k}+(A+1)^{2}=w^{2}$.

7. Expansion of $w=\sqrt{\left[A^{k}+(A+1)\right]^{2}-4 A}, A=2^{d} b, b$ odd $\geqq 1$; $d \geqq 1, k \geqq 1$. We can eliminate the case $k=1$, for then

$$
w=\sqrt{4 A^{2}+1}=[2 A, \overline{4} A] .
$$

The formulas hold ( $d$ and $b$ not both equal 1 )

$$
\left\{\begin{array}{l}
w^{2}=A^{2 k}+2(A+1) A^{k}+(A-1)^{2} ;[w]=A^{k}+A ; \\
w=A^{k}+A+r, 0<r<1 .
\end{array}\right.
$$

The reader will easily verify the following

THEOREM 7. Let $A=2^{d} b, b$ odd $\geqq 1, d \geqq 2$ be natural numbers, $w^{2}=\left[A^{k}+(A+1)\right]^{2}-4 A, k \geqq 1, w^{2}$ squarefree. The length of the primitive period in the expansion of $w$ as a periodic continued fraction equals $6(k-1)+1$. If $k$ is even $\geqq 4$, the expansion has the form

$$
\left\{\begin{array}{l}
w=\left[\overline{b_{0}, \cdots, b_{6 s+1}, b_{6 s+2}, b_{6 s+3}, b_{6 s+4}, b_{6 s+5}, b_{6 s+6}, \cdots,}\right. \\
\quad \frac{\left.b_{3 k-5}, b_{3 k-4}, 1,1, b_{3 k-4}, b_{3 k-5}, \cdots, 2 b_{0}\right]}{b_{0}=A^{k}+A}
\end{array}\right.
$$




$$
\mid \begin{aligned}
& b_{6 s+1}=1 ; b_{6 s+2}=2^{d-1} b A^{k-s-2}-1 ; b_{6 s+3}=b_{6 s+4}=1 ; \\
& \quad b_{6 s+5}=2^{d-1} b A^{s}-1 ; b_{6 s+6}=1 ; s=0,1, \cdots, \frac{1}{2}(k-4) \\
& b_{3 k-5}=1 ; b_{3 k-4}=2^{d-1} b A^{1 / 2(k-2)} .
\end{aligned}
$$

If $k$ is odd $\geqq 5$, the expansion has the form

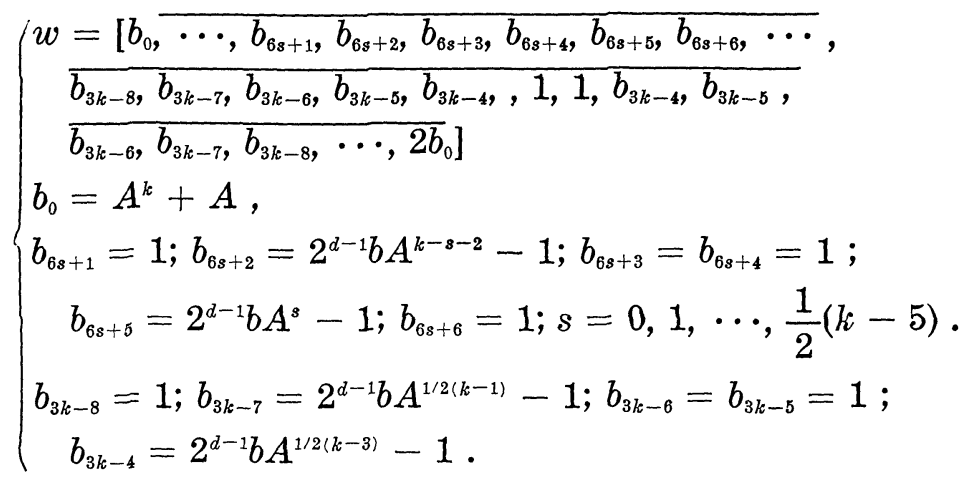

For $k=2$ we obtain the expansion

$$
\left\{\begin{array}{l}
\sqrt{\left(A^{2}+A+1\right)^{2}-4 A} \\
\left.\quad=\left[A^{2}+A, \overline{1,2^{d-1} b-1,1,1,2^{d^{-1}} b-1,1,2\left(A^{2}+A\right.}\right)\right] .
\end{array}\right.
$$

For $k=3$ we obtain the expansion

$$
\left\{\begin{array}{l}
\sqrt{\left(A^{3}+A+1\right)^{2}-4 A} \\
\quad=\left[A^{3}+A, \overline{1,2^{d-1} b A-1,1,1,2^{d-1} b-1,1,2^{d-1}-1,1,1},\right. \\
\frac{2^{d-1} b A-1,1,2\left(A^{3}+A\right)}{} .
\end{array}\right.
$$

The formula $P_{r+1}^{2}+Q_{r+1}^{2}=w^{2}$ is also verified easily, with $2 r+1=$ $6(k-1)+1$.

For $d=2, b=1, A=4$, formula (7.14) does not hold, for in this case $w^{2}=425$ and is not squarefree.

8. Expansion of $w=\sqrt{\left[A^{k}+(A+1)\right]^{2}-4 A} ; A=2^{d} b, b$ odd $\geqq$ $1 ; d \geqq 1 ; k \geqq 2$. The formulas hold (for $b$ and $d$ not both equal 1 )

$$
\left\{\begin{array}{c}
w^{2}=A^{2 k}-2(A+1) A^{k}+(A-1)^{2} ;[w]=A^{k}-A-2 ; \\
w=A^{k}-A-2+r ; 0<r<1 .
\end{array}\right.
$$

The reader will verify easily the following

THEOREM 8. Let $A=2^{d} b, b$ odd $\geqq 1, d \geqq 1$, be natural numbers, $\left.w^{2}=\left[A^{k}-A+1\right)\right]^{2}-4 A$ squarefree. If $k \geqq 4$ is even, the length of the primitive period in the expansion of $w$ as a periodic continued fraction equals $4 k-2$, and the expansion has the form 


$$
\left\{\begin{array}{l}
w=\left[b_{0}, \overline{b_{1}, b_{2}, \cdots, b_{4 s-1}, b_{4 s}, b_{48+1}, b_{4 s+2}, \cdots, 2, \cdots, b_{2(k+1)-4 s}},\right. \\
\left.\quad \overline{b_{2}(k+1)_{-4 s-1}, b_{2(k+1)-4 s-2}, b_{2(k+1)-4 s-3}, \cdots, b_{2}, b_{1}, 2 b_{0}}\right] \\
b_{0}=A^{k}-A-2 ; b_{1}=1 ; b_{2}=2^{d-1} b A^{k-2}-2 ; \\
b_{4 s-1}=2 ; b_{4 s}-2^{d-1} b A^{s-1}-1 ; b_{4 s+1}=2 ; b_{4 s+2}=2^{d-1} b A^{k-s-2}-1 ; \\
s=1,2, \cdots, \frac{1}{2}(k-2) .
\end{array}\right.
$$

For $k=2$, we obtain the expansion

$$
\left\{\begin{array}{r}
\sqrt{\left(A^{2}-A-1\right)^{2}-4 A} \\
\quad=\left[A^{2}-A-2, \overline{\left.1,2^{d-1} b-2,2,2^{d-1} b-2,1,2\left(A^{2}-A-2\right)\right]}\right. \\
(A>4)
\end{array}\right.
$$

If $k \geqq 5$ is odd, the length of the primitive period in the expansion of $w$ as a periodic continued fraction also equals $4 k-2$, and the expansion has the form

$$
\left\{\begin{array}{l}
w=\left[b_{0}, \overline{b_{1}, b_{2}, \cdots, b_{4 s-1}, b_{4 s}, b_{4 s+1}, b_{4 s+2}, \cdots, b_{2 k-3}, b_{2 k-2}, 2, b_{2 k-2}},\right. \\
\left.\overline{b_{2 k-3}, b_{2}, b_{1}, 2 b_{0}}\right] \\
b_{0}, b_{1}, b_{2}, b_{48-1}, b_{4 s}, b_{4 s+1}, b_{4 s+2} \operatorname{from}(8.13) ; s=1,2, \cdots, \frac{1}{2}(k-3) \\
b_{2 k-3}=2 ; b_{2 k-2}=2^{d-1} b A^{1 / 2(k-3)}-1 .
\end{array}\right.
$$

For $k=3$, we obtain the expansion

$$
\left\{\begin{array}{l}
\sqrt{\left(A^{3}-A-1\right)^{2}-4 A} \\
\quad=\left[A^{3}-A-2, \overline{1,2^{d-1} b A-2,2,2^{d-1} b-1,2,2^{d-1} b-1,2},\right. \\
\quad \frac{\left.2^{d-1} b A-2,1,2\left(A^{3}-A-2\right)\right]}{}
\end{array}\right.
$$

9. Expansion of $w=\sqrt{\left[(4 A)^{k}+(A-1)\right]^{2}+4 A} ; A=2^{d} b, b$ odd; $d \geqq 2$. Though, at a first glance, the structure of $w$ looks similar to that of the $\S \S 5-8$, there are surprising restrictions on the choice of $A$, and $k$. The reader will verify easily the following expansion and formulas.

$$
\begin{gathered}
\left\{\begin{array}{l}
w^{2}=(4 A)^{2 k}+2(A-1)(4 A)^{k}+(A+1)^{2} ; \\
{[w]=(4 A)^{k}+A-1 ; w=(4 A)^{k}+\mathrm{A}-1+r ; 0<r<1}
\end{array}\right. \\
w=(4 A)^{k}+A-1=\frac{1}{x_{1}} ; P_{1}=(4 A)^{k-1}+A-1 ; Q_{1}=4 A \\
\left\{\begin{array}{l}
\frac{w+(4 A)^{k}+A-1}{4 A}=2(4 A)^{k-1}+\frac{1}{x_{2}} ; \\
P_{2}=(4 A)^{k}-(A-1) ; Q_{2}=4(A-1)(4 A)^{k-1}+1 .
\end{array}\right.
\end{gathered}
$$


(9.4)

$(9.5)$

$$
\begin{gathered}
\left\{\begin{array}{l}
\frac{w+(4 A)^{k}-(A-1)}{4(A-1)(4 A)^{k-1}+1}=2+\frac{1}{x_{3}} \\
P_{3}=4(A-2)(4 A)^{k-1}+(A+1) ; Q_{3}=16(4 A)^{k-1}
\end{array}\right. \\
\left\{\begin{array}{l}
\frac{w+4(A-2)(4 A)^{k-1}+(A+1)}{16(4 A)^{k-1}} 2^{d-1} b-1+\frac{1}{x_{4}} ; \\
P_{4}=4(A-2)(4 A)^{k-1}-(A+1) ; Q_{4}=4(A-1)(4 A)^{k-1} \\
\quad+\left[A^{2}-(A+1)\right]
\end{array}\right.
\end{gathered}
$$

$$
\left\{\begin{array}{l}
\frac{w+(4 A)^{k}-8(4 A)^{k-1}-(A+1)}{(4 A)^{k}-4(4 A)^{k-1}+\left[A^{2}-(A+1)\right]}=1+\frac{1}{x_{5}} \\
P_{5}=4(4 A)^{k-1}+A^{2} ; Q_{5}=4(A+1)(4 A)^{k-1}-\left[A^{2}+(A+1)\right]
\end{array}\right.
$$

$$
\begin{gathered}
\left\{\begin{array}{l}
\frac{w+4(4 A)^{k-1}+A^{2}}{(4 A)^{k}+4(4 A)^{k-1}-\left[A^{2}+(A+1)\right]}=1+\frac{1}{x_{6}} \\
P_{6}=(4 A)^{k}-\left[2 A^{2}+(A+1)\right] ; Q_{6}=4 A^{2}
\end{array}\right. \\
\frac{w+(4 A)^{k}-\left[2 A^{2}+(A+1)\right]}{4 A^{2}}=8(4 A)^{k-2}-1+\frac{1}{x_{7}} .
\end{gathered}
$$

The reader will now verify by induction the following formulas.

$$
\begin{aligned}
& \left(\begin{array}{rl}
\text { ( i }) \quad P_{5 s+2} & =(4 A)^{k}-\left[2 A^{s+1}-(A+1)\right] ; \\
Q_{5 s+2} & =4^{s+1}\left(A^{s+1}-1\right)(4 A)^{k-s-1}-\left[A^{s+1}-(A+1)\right] ; \\
b_{5 s+2} & =2 ;
\end{array}\right. \\
& \text { (ii) } P_{5 s+3}=4^{s+1}\left(A^{s+1}-2\right)(4 A)^{k-s-1}+(A+1) \text {; } \\
& Q_{5 s+3}=4^{s+2}(4 A)^{k-s-1} ; b_{5 s+2}=2^{d-1} b A^{s}-1 ; \\
& \text { (iii) } P_{5 s+4}=4^{s+1}\left(A^{s+1}-2\right)(4 A)^{k-s-1}-(A+1) \text {; } \\
& \text { (9.9) } \\
& Q_{5 s+4}=4^{s+1}\left(A^{s+1}-1\right)(4 A)^{k-s-1}+\left[A^{s+2}-(A+1)\right] \text {; } \\
& b_{5 s+4}=1 \text {; } \\
& \text { (iv) } P_{5 s+5}=4^{s+1}(4 A)^{k-s-1}+A^{s+2} \text {; } \\
& Q_{5 s+5}=4^{s+1}\left(A^{s+1}+1\right)(4 A)^{k-s-1}-\left[A^{s+2}+(A+1)\right] ; \\
& b_{5 s+5}=1 \text {; } \\
& \text { (v) } P_{5 s+6}=(4 A)^{k}-\left[2 A^{s+2}+(A+1)\right] ; Q_{5 s+6}=4 A^{s+2} ; b_{5 s+6} \\
& =2 \cdot 4^{s+1}(4 A)^{k-s-2}-1 \text {. } \\
& s=0,1, \cdots \text {. }
\end{aligned}
$$

Formulas (9.9) are correct for $s=0$, in virtue of formulas (9.3)(9.8); then it proved that they are correct if $s$ is replaced by $s+1$. Comparing successive $P_{v}-s$, we see that the only possibility of equality is

$$
P_{5 s+2}=P_{5 s+3}
$$


This implies

$$
4^{k}=A^{2(s+1)-k} ; A=2^{d}, b=1 .
$$

From (9.11) we obtain $2 k=d[2(s+1)-k]$, hence

$$
k(d+2)=2 d(s+1) .
$$

Solving the Diophantine equation (9.12), we obtain all possible solutions as follows.

$$
\left\{\begin{array}{l}
\text { (a) } k=2 u d ; s=u(d+2)-1 ; u=1,2, \cdots ; \\
\text { (b) } k=2 u t ; d=2 t ; s=(t+1) u-1 ; t, u=1,2, \cdots ; \\
\text { (c) } k=(2 u+1) d ; s=\frac{1}{2}(d+2)(2 u+1)-1 ; d \equiv 0(\bmod 2) ; \\
\text { (d) } 2 k=(2 u+1) d ; 4 s=(d+2)(2 u+1)-4 ; d \equiv 2(\bmod 4) .
\end{array}\right.
$$

The reader should note the following procedure: after $k$ and $d$ have been chosen from (9.13), (a)-(d), $s$ is a function of $k, d$; for constant $k_{0}, d_{0}$ we shall denote

$$
s_{0}=F\left(k_{0}, d_{0}\right) .
$$

The length of the primitive period in the expansion of $w$ for any choice of $(k, d)$ from $(9.13)$ then becomes $m=10 s_{0}+4$.

Comparing successive $Q_{v}-s$, we see that the only possibility of equality is

$$
Q_{5 s+4}=Q_{5 s+5}
$$

This implies

$$
4^{k}=A^{2 s+3-k} ; A=2^{d} ; b=1 .
$$

From (9.16) we obtain $2 k=d(2 s+3-1)$, hence

$$
k(d+2)=d(2 s+3) .
$$

Solving the Diophantine equation (9.17), we obtain all possible solutions as follows

$$
\left\{\begin{array}{l}
(\text { a) } \quad k=u d, 2 s+3=u(2+d), u, d \equiv 1(2) ; \\
\text { (b) } k=u t ; d=2 t ; 2 s+3=u(t+1) ; u \equiv 1(2) ; t \equiv 0(2) .
\end{array}\right.
$$

We again denote $s_{0}=F\left(k_{0}, d_{0}\right)$, for any choice of fixed $k$ and $d$ from (9.18). The length of the primitive period in the expansion of $w$ for anyc hoice of $(k, d)$ from $(9.18)$ then becomes $m=10 s_{0}+g$. For $d=1,(b=1)$, we obtain $w=\sqrt{\left(8^{k}+1\right)^{2}+8}$, which is easily expanded and is left to the reader. We can now state. 
Theorem 9. Let $A=2^{d}, d \geqq 2, w^{2}=\left[(4 A)^{k}+(A-1)\right]^{2}+4 A$ squarefree. If $\left(k_{0}, d_{0}, s_{0}\right)$ is any solution vector of the Diophantine equation (9.12), given by the value Table (9.13), (a)-(d), then the primitive period in the expansion of $w$ as a periodic continued fraction has length $m=10_{s_{0}}+4,\left(s_{0} \geqq 1\right)$, and the form

$$
\left\{\begin{array}{l}
w=\left[b_{0}, \overline{b_{1}, \cdots, b_{5 s+2}, b_{5 s+3}, b_{5 s+4}, b_{5 s+5}, b_{5 s+6}, \cdots, 2, \cdots, b_{5\left(s_{0}-s\right)+1}},\right. \\
\left.\quad \overline{b_{5\left(s_{0}-s\right)}, b_{5\left(s_{0}-s\right)-1}, b_{5\left(s_{0}-s\right)-2}, b_{5\left(s_{0}-s\right)-3}, \cdots, b_{1}, 2 b_{0}}\right] \\
b_{0}=(4 A)^{k_{0}}+A-1 ; b_{1}=2(4 A)^{k_{0}-1} ; \\
b_{5 s+2}=2 ; b_{5 s+3}=2^{d_{0}-1} A^{s}-1 ; b_{5 s+4}=b_{5 s+5}=1 ; \\
b_{5 s+6}=2 \cdot 4^{s+1}(4 A)^{k_{0}-s-2}-1 . \quad s=0,1, \cdots, s_{0}-1 .
\end{array}\right.
$$

If $\left(k_{0}, s_{0}, d_{0}\right)$ is any solution vector of the Diophantine equation (9.17), given by the value table (9.18), (a), (b), then the primitive period in the expansion of $w$ as a periodic continued fraction has length $m=10 s_{0}+9$, and the form

$$
\left\{\begin{array}{l}
w=\left[b_{0}, \overline{b_{1}, \cdots, b_{5 s+2}, b_{5 s+3}, b_{5 s+4}, b_{5 s+5}, b_{5 s+6}, \cdots, 2,2^{d_{0}-1} b A^{s_{0}}-1}\right. \\
\left.\quad \overline{1,1,2^{d_{0}-1} b A^{s_{0}}-1,2, \cdots, b_{1}, 2 b_{0}}\right] \\
\text { where the } b_{0}, b_{1}, b_{5 s+2}, \cdots, b_{5 s+8} \text { are the same as in }(9.19) \\
s=0,1, \cdots, s_{0}-1 .
\end{array}\right.
$$

10. Exapansion of $w=\sqrt{\left[(4 A)^{k}+A+1\right]^{2}-4 A} ; A=2^{d} b, b$ odd, $d \geqq 2$. The reader will verify easily the following formulas and expansions

(10.1) $\left\{\begin{array}{l}w^{2}=(4 A)^{2 k}+2(A+1)(4 A)^{k}+(A-1)^{2} ;[w]=(4 A)^{k}+A ; \\ w=(4 A)^{k}+A+r ; 0<r<1 .\end{array}\right.$
(10.2) $w=(4 A)^{k}+A+\frac{1}{x_{1}} ; P_{1}=(4 A)^{k}+A ; Q_{1}=2(4 A)^{k}-(2 A-1)$.

$$
\frac{w+(4 A)^{k}+A}{2(4 A)^{k}-(2 A-1)}=1+\frac{1}{x_{2}} ; P_{2}=(4 A)^{k}-(3 A-1) ; Q_{2}=4 A
$$

$$
\begin{gathered}
\left\{\begin{array}{l}
\frac{w+(4 A)^{k}-(3 A-1)}{4 A}=2(4 A)^{k-1}-1+\frac{1}{x_{3}} \\
P_{3}=(4 A)^{k}-(A+1) ; Q_{3}=4(A+1)(4 A)^{k-1}-1
\end{array}\right. \\
\left\{\begin{array}{l}
\frac{w+(4 A)^{k}-(A+1)}{(4 A)^{k}+4(4 A)^{k-1}-1}=1+\frac{1}{x_{4}} \\
P_{4}=4(4 A)^{k-1}+A ; Q_{4}=4(A-1)(4 A)^{k-1}+(2 A-1)
\end{array}\right.
\end{gathered}
$$

$$
\left\{\begin{array}{l}
\frac{w+4(4 A)^{k-1}+A}{(4 A)^{k}-4(4 A)^{k-1}+2 A-1}=1+\frac{1}{x_{5}} ; \\
P_{5}=4(A-2)(4 A)^{k-1}+A-1 ; Q_{5}=16(4 A)^{k-1} .
\end{array}\right.
$$


(10.7)

$$
\left\{\begin{array}{l}
\frac{w+4(A-2)(4 A)^{k-1}+A-1}{16(4 A)^{k-1}}=2^{d-1} b-1+\frac{1}{x_{6}} \\
P_{6}=4(A-2)(4 A)^{k-1}-(A-1) ; Q_{6}=4(A-1)(4 A)^{k-1} \\
\quad+\left[A^{2}-(A-1)\right] .
\end{array}\right.
$$

(10.8) $\left\{\frac{w+(4 A)^{k}-8(4 A)^{k-1}-(A-1)}{(4 A)^{k}-4(4 A)^{k-1}+\left[A^{2}-(A-1)\right]}=1+\frac{1}{x_{7}}\right.$

$$
P_{7}=4(4 A)^{k-1}+A^{2} ; Q_{7}=4(A+1)(4 A)^{k-1}-\left[A^{2}+(A-1)\right] \text {. }
$$

$$
\left\{\begin{array}{l}
\frac{w+4(4 A)^{k-1}+A^{2}}{(4 A)^{k}+4(4 A)^{k-1}-\left[A^{2}+(A-1)\right]}=1+\frac{1}{x_{8}} \\
P_{8}=(4 A)^{k}-\left[2 A^{2}+(A-1)\right] ; Q_{8}=4 A^{2} .
\end{array}\right.
$$

We now prove by induction the following formulas as before; they are correct for the parameter $s=0$, and it is then proved that they are correct for substituting $s$ by $s+1$ :

(10.10)

$$
\begin{aligned}
& \text { ( ( i ) } \quad P_{6 s+2}=(4 A)^{k}-\left[2 A^{s+1}+(A-1)\right] ; Q_{6 s+2}=4 A^{s+1} ; b_{6 s+2} \\
& =2 \cdot 4^{s}(4 A)^{k-s-1}-1 \text {; } \\
& \text { (ii) } P_{6 s+3}=(4 \mathrm{~A})^{k}-\left[2 A^{s+1}-(A-1)\right] \text {; } \\
& Q_{6 s+3}=4^{s+1}\left(A^{s+1}+1\right)(4 A)^{k-s-1}-\left[A^{s+1}-(A-1)\right] \text {; } \\
& b_{6 s+3}=1 \text {; } \\
& \text { (iii) } P_{6 s+4}=4^{s+1}(4 A)^{k-s-1}+A^{s+1} \text {; } \\
& Q_{6 s+4}=4^{s+1}\left(A^{s+1}-1\right)(4 A)^{k-s-1}+\left[A^{s+1}+(A-1) ;\right. \\
& b_{6 s+4}=1 \text {; } \\
& \text { (iv) } P_{6 s+5}=4^{s+1}\left(A^{s+2}-2\right)(4 A)^{k-s-1}+A-1 \text {; } \\
& Q_{6 s+5}=4^{s+2}(4 A)^{k-s-1} ; b_{6 s+5}=2^{d-1} b A^{s}-1 ; \\
& \text { (v) } P_{6 s+6}=4^{s+1}\left(A^{s+1}-2\right)(4 A)^{k-s-1}-(A-1) \text {; } \\
& Q_{6 s+6}=4^{s+1}\left(A^{s+1}-1\right)(4 A)^{k-s-1}+\left[A^{s+2}-(A-1)\right] \text {; } \\
& b_{6 s+6}=1 \text {; } \\
& \text { (vi) } P_{6 s+7}=4^{s+1}(4 A)^{k-s-1}+A^{s+2} \text {; } \\
& Q_{6 s+7}=4^{s+1}\left(A^{s+1}+1\right)(4 A)^{k-s-1}-\left[A^{s+2}+(A-1)\right] ; \\
& b_{6 s+7}=1 ; s=0,1, \cdots \text {. }
\end{aligned}
$$

Comparing successive $P_{v}-s$ and $Q_{v}-s$, we obtain the cases

$$
Q_{6 s+3}=Q_{6 s+4} \text {. }
$$

This implies

$$
4^{k}=A^{2(s+1)-k} ; A=2^{d} ; b=1 .
$$

From (10.12) we obtain $2 k=d[2(s+1)-k]$, 


$$
(d+2) k=2 d(s+1) .
$$

(10.13) is the same equation as (9.12) and its solutions are given by (9.13). If $\left(k_{0}, d_{0}, s_{0}\right)$ is a solution vector of (10.13), then the length of the primitive period in the expansion of $w$ equals $m=10 s_{0}+7$.

$$
Q_{6 s+6}=Q_{6 s+7} \text {. }
$$

This implies

$$
4^{k}=A^{2 s+3-k} ; A=2^{d} ; b=1 .
$$

From (10.15) we obtain $2 k=d(2 s+3-k)$,

$$
k(d+2)=d(2 s+3) .
$$

Equation (10.16) is equation (9.17), and its solutions are given by (9.18), (a), (b). If $\left(k_{0}, d_{0}, s_{0}\right)$ is a solution vector of (10.16), then the length of the primitive period in the expansion of $w$ equals $m=$ $10 s_{0}+13$. The restriction on $d \geqq 2$ results from the value of $b_{5}=$ $2^{d-1}-1$ in (10.7). If $d=1$, we would have $b_{5}=0$, which is impossible. The case $d=1, A=2$, yields $w=\sqrt{\left(8^{k}+3\right)^{2}-8}$, and the expansion of this $w$ is left to the reader. We can now state.

TheOREM 10. Let $A=2^{d}, d \geqq 2, w^{2}=\left[(4 A)^{k}+(A+1)\right]^{2}-4 A$ squarefree. If $\left(k_{0}, d_{0}, s_{0}\right)$ is any solution vector of the Diophantine equation (10.13), given by the value table (9.13), (a)-(d), then the primitive period in the expansion of $w$ as a periodic continued fraction has length $m=10 s_{0}+7$, and the form

$$
\left\{\begin{array}{l}
w=\left[b_{0}, \overline{b_{1}, \cdots, b_{6 s+2}, b_{6 s+3}, b_{6 s+4}, b_{6 s+5}, b_{6 s+6}, b_{6 s+7}, \cdots, \cdots}\right. \\
\left.\frac{2 \cdot 4^{s}(4 A)^{k_{0}-s_{0}-1}-1,1,1,2 \cdot 4^{s}(4 A)^{k_{0}-s_{0}-1}, \cdots, b_{1}, 2 b_{0}}{}\right] \\
b_{0}=(4 A)^{k_{0}}+A ; b_{1}=1 \\
b_{6 s+2}=2 \cdot 4^{s}(4 A)^{k_{0}-s-1}-1 ; b_{6 s+3}=b_{6 s+4}=1 ; \\
b_{6 s+5}=2^{d_{0}-1} A^{s}-1 ; b_{6 s+6}=b_{6 s+7}=1 .
\end{array}\right.
$$

If $\left(k_{0}, d_{0}, s_{0}\right)$ is any solution vector of the Diophantine equation (10.16), given by the value table (9.18), (a), (b), then the primitive period in the expansion of $w$ as a periodic continued fraction has length $m=10 s_{0}+13$, and the form

$$
\left\{\begin{array}{l}
w=\left[b_{0}, \widehat{b_{1}, \cdots, b_{6 s+2}, b_{6 s+3}, b_{6 s+4}, b_{6 s+5}, b_{6 s+6}, b_{6 s+7}, \cdots},\right. \\
\left.\frac{2 \cdot 4^{s_{0}}(4 A)^{k_{0}-s_{0}-1}-1,1,1,2^{d_{0}-1} A^{s_{0}}-1,1,1,2^{d_{0}-1} A^{s_{0}}-1}{1,1,2 \cdot 4^{s_{0}}(4 A)^{k_{0}-s_{0}-1}-1, \cdots, b_{1}, 2} b_{0}\right] \\
b_{0}, b_{1}, b_{6 s+2}, \cdots, b_{6 s+7} \text { from }(10.17), s=0,1, \cdots, s_{0}-1
\end{array}\right.
$$

11. Expansion of $w=\sqrt{\left[A^{k}+(A-1)\right]^{2}+4 A} ; A=2^{d} b+1 ; d \geqq$ 
$1 ; b$ odd. This expansion is remarkable in the sense that the cycles of the period are of length eleven; so are the cycles in the next two sections, while in the last section the length of the cycle is even twelve.

We obtain the formulas

$$
\begin{aligned}
w^{2} & =A^{2 k}+2(A-1) A^{k}+(A+1)^{2} ;[w]=A^{k}+A-1 ; w \\
& =A^{k}+A-1+r ; 0<r<1 .
\end{aligned}
$$

The reader will easily verify the following:

TheOREM 11. Let $A=2^{d} b+1, d \geqq 1, b$ odd, $w^{2}=\left[A^{k}+(A-1)\right]^{2}+$ $4 A$ squarefree; let further $k \equiv 4(\bmod 6), k \geqq 4, s_{0}=(1 / 6)(k-4)$. Then the length of the primitive period in the expansion of $w$ as a periodic continued fraction equals $1 / 3(11 k-14)$, and the expansion has the form, for $k \geqq 10$,

$$
\left\{\begin{array}{l}
w=\left[b_{0}, \overline{b_{1}, \cdots, b_{11 s+2}, b_{11 s+3}, \cdots, b_{11 s+12}, \cdots, b_{11 s_{0}+2}, b_{11 s_{0}+3}, b_{11 s_{0}+4}},\right. \\
\quad \overline{\left.2, b_{11 s_{0}+4}, b_{11 s_{0}+3}, b_{11 s_{0}+2}, \cdots, b_{1}, 2 b_{0}\right]} \\
b_{0}=A^{k}+A-1 ; b_{1}=2^{d-1} b A_{k-2} ; b_{11 s_{0}+2}=1 ; b_{11 s_{0}+3}=2 A^{1 / 2(k-2)}-1 ; \\
b_{11 s_{0}+4}={ }^{d-1} b A_{1 / 2 k-2} ; b_{11 s+2}=1 ; b_{11 s+3}=2 A^{3 s+1}-1 ; \\
b_{11 s+4}=2^{d-1} b A_{k-3(s+1)} ; b_{11 s+5}=2 ; b_{11 s+6}=2^{d-1} b A_{3 s+1} ; \\
b_{11 s+7}=2 A^{k-3(s+1)}-1 ; b_{11 s+8}=1 ; b_{11 s+9}=2^{d-1} b A_{3 s+2} ; \\
b_{11 s+10}=b_{11 s+1}-1 ; b_{11 s+12}=2^{d-1} b A_{k-3(s+1)-2}-2 ; \\
s=0,1, \cdots, s_{0}-1 ; A_{u}=(A-1)^{-1}\left(A^{u+1}-1\right) ; u=0,1, \cdots ; \\
A_{0}=1 .
\end{array}\right.
$$

If $k=4$, the expansion of $w$ has the form

$$
\left\{\begin{array}{l}
\frac{\sqrt{\left[A^{4}+(A-1)\right]^{2}+4 A}=\left[A^{4}+A-1, \overline{2^{d-1} b\left(A^{2}+A+1\right), 1}\right.}{2 A-1,2^{d-1} b, 2,2^{d-1} b, 2 A-1,1,2^{d-1} b\left(A^{2}+A+1\right)} \\
\overline{\left.2\left(A^{4}+A-1\right)\right]}
\end{array}\right.
$$

If $k=3$, the expansion has the form

$$
\left\{\begin{array}{l}
\sqrt{\left[A^{3}+(A-1)\right]^{2}+4 A}=\left[b_{0}, b_{1}, \cdots, b_{12}, b_{13}, b_{14}, b_{12}, \cdots, b_{1}, 2 b_{0}\right] \\
b_{0}=A^{3}+A-1 ; b_{1}=2^{d-1} b(A+1) ; b_{2}=1 ; b_{3}=2 A-1 ; \\
b_{4}=2^{d-1} b ; b_{5}=2 ; b_{6}=2^{d-1} b(A+1) ; b_{7}=b_{8}=1 ; \\
b_{9}=2^{d-1} b\left(A^{2}+A\right)+A-1 ; b_{10}=2 A^{2}+1 ; b_{11}=1 ; \\
\quad b_{12}=2^{d-1} b-1 ; b_{13}=b_{14}=1 .
\end{array}\right.
$$

The length of the primitive period in the expansion of $w$ for $k=3$ equals 27 . Let further be $k \equiv 1(\bmod 6), k \geqq 7, s_{0}=(1 / 6)(k-7)$. Then the length of the period in the expansion of $w$ as a periodic continued fraction equals $1 / 3(11 k-14)$, and the expansion has the form, for $k \geqq 13$, 
(11.5)

$$
\left\{\begin{array}{l}
w=\left[b_{0}, \overline{b_{1}, \cdots, b_{11 s+2}, b_{11 s+3}, \cdots, b_{11 s+12}, \cdots, b_{11 s_{0}+2}, b_{11 s_{0}+3}, \cdots},\right. \\
\left.\quad \overline{b_{11 s_{0}+9}, 1,1, b_{11 s_{0}+9}, b_{1 s_{0}+8}, \cdots, b_{11 s_{0}+2}, \cdots, b_{1}, 2 b_{0}}\right] \\
b_{0}=A^{k+} A-1 ; b_{1}=2^{d-1} b A_{k-2} ; b_{11 s_{0}+2}=1 ; b_{11 s_{0}+3}=2 A^{1 / 2(k-s)}-1 ; \\
b_{11 s_{0}+4}=2^{d-1} b A_{1 / 2(k+1)} ; b_{11 s_{0}+5}=2 ; b_{11 s_{0}+6}=2^{d-1} b A_{1 / 2(k-5)} ; \\
b_{11 s_{0}+7}=2 A^{1 / 2(k+1)}-1 ; b_{11 s_{0}+8}=1 ; \\
b_{11 s_{0}+9}=2^{d-1} b A_{1 / 2(k-3)}-1 ; \\
b_{11 s+2}, b_{11 s+3}, \cdots, b_{11 s+12} \text { as in }(11.19) ; s=0,1, s_{0}-1 .
\end{array}\right.
$$

It is left to the reader to find the expansion of $w$ for the cases $k=5,6,7$.

12. Expansion of $w=\sqrt{\left[A^{k}-(A-1)\right]^{2}+4 A} ; A=2^{d} b+1 ; d \mathbb{N}$ $1 ; b$ odd. We use the notation, as before,

$$
\begin{aligned}
A^{u+1}-1 & =(A-1) A_{u} ; u=0,1, \cdots ; A_{0} \\
& =1 ; \frac{1}{2}\left(A^{u+1}-1\right)=2^{d-1} b A_{u} .
\end{aligned}
$$

The following formulas hold:

$$
\left\{\begin{array}{l}
w^{2}=A^{2 k}-2(A-1) A^{k}+(A+1)^{2} ;[w]=A^{k}-A+1 \\
w=A^{k}-A+1+r, 0<r<1
\end{array}\right.
$$

The reader will easily verify the following

Theorem 12. Let $A=2^{d} b+1, d \geqq 1, b$ odd, $w^{2}=\left[A^{k}-(A-1)\right]^{2}+$ $4 A$ squarefree; let further $k \equiv 1(\bmod 6), k \geqq 7, s_{0}=(1 / 6)(k-7)$. Then the length of the primitive period in the expansion of $w$ as a periodic continued fraction equals 11/3(k-1), and the expansion has the form, for $k \geqq 13$,

$$
\left\{\begin{array}{l}
w=\left[b_{0}, \cdots, b_{11 s+1}, b_{11 s+2}, \cdots, b_{11 s+11}, \cdots, b_{11 s_{0}+1}, b_{11 s_{0}+2}, \cdots,\right. \\
\quad \overline{\left.b_{11 s_{0}+1}, 2, b_{11 s 0+10}, b_{11 s_{0}+9}, \cdots, b_{11 s_{0}+1}, \cdots, 2 b_{0}\right]} \\
b_{11 s+1}=2^{d-1} b A_{k-3 s-2} ; b_{11 s+2}=2 A^{3 s+1}-1 ; \\
b_{11 s+3}=b_{11 s+5}=b_{11 s+6}=b_{11 s+8}=1 ; b_{11 s+4}=2^{d-1} b A_{k-3(s+1)}-1 ; \\
b_{11 s+7}=2^{d-1} b A_{3 s+1}-1 ; b_{11 s+9}=2 A^{k-3(s+1)}-1 ; \\
b_{11 s+10}=2^{d-1} b A_{3 s+2} ; b_{0}=A^{k}-A+1 ; b_{11 s_{0}+1}=2^{d-1} b A_{1 / 2(l)+3)} ; \\
b_{1 s_{0}+2}=2 A^{1 / 2(k-5)}-1 ; b_{11 s_{0}+5}=b_{11 s_{0}+6}=b_{11 s_{0}+8}=1 ; \\
b_{1 s_{0}+4}=2^{d-1} b A_{1 / 2(k+1)}-1 ; b_{11 s_{0}+7}=2^{d-1} b A_{1 / 2(k-5)}-1 ; \\
b_{1 s_{0}+9}=2 A^{1 / 2(k+1)}-1 ; b_{11 s_{0}+10}=2^{d-1} b A_{1 / 2(k-3)} ; b_{11 s+11}=2 . \\
s=0,1, \cdots, s_{0}-1 ; A_{u}=(A-1)^{-1}\left(A^{u+1}-1\right), u=0,1, \cdots ; \\
A_{0}=1 .
\end{array}\right.
$$


For $k=7$, the expansion has the form

$$
\left\{\begin{array}{l}
\sqrt{\left[A^{7}-(A-1)\right]^{2}+4 A}=\left[b_{0}, \overline{b_{1}, b_{2}, \cdots, b_{10}, 2, b_{10}, \cdots, b_{2}, b_{1}, 2 b_{0}}\right] \\
b_{0}=A^{7}-A+1 ; b_{1}=2^{d-1} b A_{5} ; b_{2}=2 A-1 ; \\
b_{3}=b_{5}=b_{6}=b_{8}=1 ; b_{4}=2^{d-1} b A_{4}-1 ; b_{7}=2^{d-1} b A_{1}-1 ; \\
b_{9}=2 A^{4}-1 ; b_{10}=2^{d-1} b A_{2} .
\end{array}\right.
$$

Let further be $k \equiv 4(\bmod 6) ; s_{0}=(1 / 6)(k-4) ; k \geqq 4$. Then the length of the primitive period in the expansion of $w$ equals $11 / 3(k-1)$, and the expansion has the form, for $k \geqq 10$,

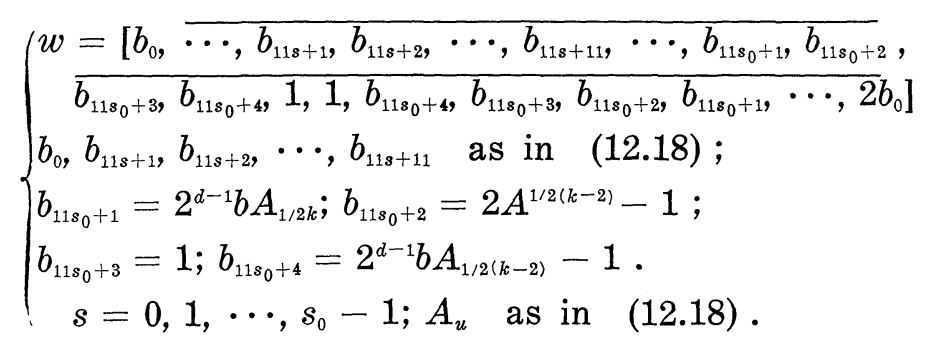

For $k=4$, the expansion has the form

$$
\left\{\begin{array}{l}
\sqrt{\left[A^{4}-(A-1)\right]^{2}+4 A}=\left[b_{0}, \overline{\left.b_{1}, b_{2}, b_{3}, b_{4}, 1,1, b_{4}, b_{3}, b_{2}, b_{1}, 2 b_{0}\right]}\right. \\
b_{0}=A^{4}-A+1 ; b_{1}=2^{d-1} b A_{2} ; b_{2}=2 A-1 ; b_{3}=1 \\
b_{4}=2^{d-1} b A_{1}-1
\end{array}\right.
$$

13. Expansion of $w \sqrt{\left(A^{k}+A+1\right)^{2}-4 A ;} A=2^{d} b+1 ; d \geqq 1 ; b$ odd.

TheOREM 13. Let $A=2^{d} b+1, d \geqq 1, b$ odd,$w^{2}=\left(A^{k}+A+1\right)^{2}-$ $4 A$ squarefree; let further $k \equiv 4(\bmod 6), k \geqq 4 ; s_{0}=1 / 6(k-4)$. Then the length of the primitive period in the expansion of $w$ as a continued fraction equals $1 / 3(10 k-7)$, and the expansion has the form, for $k \geqq 10$,

$$
\left\{\begin{array}{l}
w=\left[b_{0}, \overline{b_{1}, \cdots, b_{10 s+2}, b_{10 s+3}, \cdots, b_{10 s+11}, \cdots, b_{10 s_{0}+2}, b_{10 s_{0}+3}, b_{10 s_{0}+4}},\right. \\
\left.\quad \overline{1,1, b_{10 s_{0}+4}, b_{10 s_{0}}+3, b_{10 s_{0}+2}, \cdots, b_{1}, 2 b_{0}}\right] \\
b_{0}=A^{k}+A ; b_{1}=1 ; b_{11 s_{0}+2}=2^{d-1} b A_{1 / 2 k} ; b_{10 s_{0}+3}=2 A^{1 / 2(k-2)} ; \\
b_{10 s_{0}+4}=2^{d-1} b A_{1 / 2(k-2)} ; b_{10 s+2}=2^{d-1} b A_{k-3 s-2} ; \\
b_{10 s+3}=2 A^{3 s+1} ; b_{10 s+4}=2^{d-1} b A_{k-3(s+1)} ; \\
b_{10 s+5}=b_{10 s+6}=b_{10 s+10}=b_{10 s+11}=1 ; b_{10 s+7}=2^{d-1} b A_{3 s+1} ; \\
b_{10 s+8}=2 A^{k-3(s+1)} ; b_{10 s+9}=2^{d-1} b A_{3 s+2} ; \\
\quad s=0,1, \cdots, s_{0}-1 ; A^{u+1}-1=(A-1) A_{u} ; u=1,2, \cdots .
\end{array}\right.
$$

For $k=4$, the expansion has the form 
(13.2)

$$
\left\{\begin{array}{l}
\sqrt{\left(A^{4}+A+1\right)^{2}-4 A}=\left[b_{0}, \overline{b_{1}, b_{2}, b_{3}, b_{4}, 1,1, b_{4}, b_{3}, b_{2}, b_{1}, 2 b_{0}}\right] \\
b_{0}=A^{4}+A ; b_{1}=1 ; b_{2}=2^{d-1} b A_{2} ; b_{3}=2 A ; b_{5}=2^{d-1} b(A+1)
\end{array} .\right.
$$

Let further $k \equiv 1(\bmod 6), k \geqq 7, s_{0}=(1 / 6)(k-7)$. Then the length of the primitive period in the expansion of $w$ as a periodic continued fraction equals $1 / 3(10 k-7)$, and the expansion has the form, for $k \geqq 13$,

$$
\left\{\begin{array}{l}
w=\left[b_{0}, \overline{b_{1}, \cdots, b_{10 s+2},}, b_{10 s+3}, \cdots, b_{10 s+11}, \cdots, b_{10 s_{0}+2}, b_{10 s_{0}+3}, \cdots,\right. \\
\quad \overline{\left.b_{10 s_{0}+9}, 1,1, b_{10 s_{0}+9}, b_{10 s_{0}+8}, \cdots, b_{10 s_{0}+2}, \cdots, b_{1}, 2 b_{0}\right]} \\
b_{0}, b_{1}, b_{10 s+2}, b_{10 s+2}, \cdots, b_{10 s+11} \text { as in }(13.17), s=0,, 1 \cdots, \\
s_{0}-1 ; b_{10 s_{0}+2}=2^{d-1} b A_{1 / 2(k+3)} ; b_{10 s_{0}+3}=2 A^{1 / 2(k-5)} ; \\
b_{10 s_{0}+4}=2^{d-1} b A_{1 / 2(k+1)} ; b_{10 s_{0}+5}=b_{10 s_{0}+6}=1 ; \\
b_{10 s_{0}+7}=2^{d-1} b A_{1 / 2(k-5)} ; b_{10 s_{0}+8}=2 A^{1 / 1(k+1)} ; \\
b_{10 s_{0}+9}=2^{d-1} b A_{1 / 2(k-3)} .
\end{array}\right.
$$

For $k=7$, the expansion of $w$ has the form

$$
\left\{\begin{array}{l}
\sqrt{\left(A^{7}+A+1\right)^{2}-4 A}=\left[b_{0}, b_{1}, \overline{b_{2}, \cdots, b_{9}, 1,1, b_{9}, \cdots, b_{2}, b_{1}, 2 b_{0}}\right] \\
b_{0}=A^{7}+A ; b_{1}=1 ; b_{2}=2^{d-1} b A_{5} ; b_{3}=2 A ; b_{4}=2^{d-1} b A_{4} \\
b_{5}=b_{6}=1 ; b_{7}=2^{d-1} b A_{1} ; b_{8}=2 A^{4} ; b_{9}=2^{d-1} b A_{2} .
\end{array}\right.
$$

14. Expansion of $w=\sqrt{\left[A^{k}-(A+1)\right]^{2}-4 A} ; A=2^{d} b+1 ; d \geqq$ $1 ; b \geqq 1$ odd. This case is the most interesting of all treated in this part of the paper, since the length of the cycle is greater than any previous one, namely 12 . With the previous notation, $A^{u+1}-1=$ $(A-1) A_{u}, u=0,1, \cdots ; A_{0}=1$, the reader will easily verify the following expansions and formulas.

$$
\left\{\begin{array}{l}
w^{2}=A^{2 k}-2(A+1) A^{k}+(A-1)^{2} ;[w]=A^{k}-A-2 \\
w=A^{k}-A-2+r, 0<r<1
\end{array}\right.
$$

$$
\left\{\begin{array}{l}
\frac{w+A^{k}-5 A-1}{4 A}=2^{d-1} b A_{k-2}-2+\frac{1}{x_{3}} ; P_{3}=A^{k}-(5 A-1) \\
Q_{3}=(2 A-1) A^{k-1}-2(3 A-1) .
\end{array}\right.
$$

(14.5) $\left\{\begin{array}{l}\frac{w+A^{k}-5 A+1}{2 A^{k}-A^{k-1}-6 A+2}=1+\frac{1}{x_{4}} ; P_{4}=(A-1) A^{k-1}-(A-1) \text {; } \\ Q_{4}=A^{k-1}\end{array}\right.$ 
(14.6)

$$
\begin{aligned}
& \frac{w+A^{k}-A^{k-1}-A+1}{A^{k-1}} 2=(A-1)+\frac{1}{x_{5}} ; \\
& P_{5}=(A-1) A^{k-1}+(A-1) ; Q_{5}=(2 A-1) A^{k-1}-2\left[2 A^{2}-(A-1)\right] .
\end{aligned}
$$

$$
\left\{\begin{array}{c}
\frac{w+A^{k}-A^{k-1}+A-1}{2 A^{k}-A^{k-1}-2\left[2 A^{2}-(A-1)\right]}=1+\frac{1}{x_{6}} ; \\
P_{6}=A^{k}-\left[4 A^{2}-(A-1)\right] ; Q_{6}=4 A^{2} .
\end{array}\right.
$$

(14.8)

$$
\left\{\begin{array}{l}
\frac{w+A^{k}-4 A^{2}+A-1}{4 A^{2}}=2^{d-1} b A_{k-3}-1+\frac{1}{x_{7}} ; \\
P_{7}=A^{k}-\left[2 A^{2}+(A-1)\right] ; Q_{7}=\left(A^{2}-1\right) A^{k-2}-\left[A^{2}+(A-1)\right] ;
\end{array}\right.
$$

$$
\left\{\begin{array}{l}
\frac{w+A^{k}-2 A^{2}-A+1}{A^{k}-A^{k-2}-A^{2}-A+1}=2+\frac{1}{x_{8}} ; \\
\quad P_{8}=\left(A^{2}-2\right) A^{k-2}-(A-1) ; Q_{8}=4 A^{k-2} ;
\end{array}\right.
$$

(14.10) $\left\{\begin{array}{l}\frac{w+A^{k}-2 A^{k-2}-(A-1)}{4 A^{k-2}} \\ =2^{d-1} b A_{1}-1+\frac{1}{x_{9}} ; P_{9}=\left(A^{2}-4\right) A^{k-2}+(A-1) ; Q_{9} \\ =2\left(A^{2}-2\right) A^{k-2}-\left[A^{3}-2(A-1)\right] ;\end{array}\right.$

$$
\frac{w+A^{k}-4 A^{k-2}+(A-1)}{2 A^{k}-4 A^{k-2}-A^{3}+2(A-1)}
$$

$$
=1+\frac{1}{x_{10}} ; P_{10}=A^{k}-\left[A^{3}-(A-1)\right] ; Q_{10}=A^{3} ;
$$

(14.12)

$$
\left\{\begin{array}{l}
\frac{w+A^{k}-A^{3}+A-1}{A^{3}}=2 A^{k-3}-2+\frac{1}{x_{11}} \\
P_{11}=A^{k}-\left[A^{3}+(A-1)\right] \\
Q_{11}=2\left(A^{3}-2\right) A^{k-3}-\left[A^{3}+2(A-1)\right]
\end{array}\right.
$$

(14.13)

(14.14) $\left\{\begin{array}{l}\frac{w+A^{k}-4 A^{k-3}-(A-1)=}{4 A^{k-3}}=2^{d-1} b A_{2}-1+\frac{1}{x_{13}} ; \\ P_{13}=\left(A^{3}-2\right) A^{k-3}+(A-1) ; \\ Q_{13}=\left(A^{3}-1\right) A^{k-3}-\left[A^{4}-(A-1)\right] ;\end{array}\right.$

$$
\left\{\begin{array}{l}
\frac{w+A^{k}-A^{3}-(A-1)}{2 A^{k}-4 A^{k-3}-A^{3}-2(A-1)}=1+\frac{1}{x_{12}} ; \\
P_{12}=\left(A^{3}-4\right) A^{k-3}-(A-1) ; Q_{12}=4 A^{k-3} ;
\end{array}\right.
$$

$$
\left\{\begin{array}{l}
\frac{w+A^{k}-2 A^{k-3}+(A-1)}{A^{k}-A^{k-3}-A^{4}+(A-1)}=2+\frac{1}{x_{14}} ; \\
P_{14}=A^{k}-\left[2 A^{4}-(A-1)\right] ; Q_{14}=4 A^{4} ;
\end{array}\right.
$$


(14.16)

$$
\frac{w+A^{k}-2 A^{4}+(A-1)}{4 A^{4}} 2 d^{-1} b A_{k-5}-1+\frac{1}{x_{15}} .
$$

We now prove the formulas:

$$
\left\{\begin{array}{l}
P_{12 s+3}=A^{k}-\left[4 A^{3 s+1}+(A-1)\right] ; \\
Q_{12 s+3}=\left(2 A^{3 s+1}-1\right) A^{k-3 s-1}-2\left[2 A^{3 s+1}+(A-1)\right] ; b_{12 s+3}=1 ; \\
P_{12 s+4}=\left(A^{3 s+1}-1\right) A^{k-3 s-1}-(A-1) ; \\
Q_{12 s+4}=A^{k-3 s-1} ; b_{12 s+4}=2 A^{3 s+1}-2 ; \\
P_{12 s+5}=\left(A^{3 s+1}-1\right) A^{k-3 s-1}+(A-1) ; \\
Q_{12 s+5}=\left(2 A^{3 s+1}-1\right) A^{k-3 s-1}-2\left[2 A^{3 s+2}-(A-1)\right] ; b_{12 s+5}=1 ; \\
P_{12 s+6}=A^{k}-\left[4 A^{3+2}-(A-1)\right] ; \\
Q_{12 s+6}=4 A^{3 s+2} ; b_{12 s+6}=2^{d-1} b A_{k-3(s+1)}-1 ; \\
P_{12 s+7}=A^{k}-\left[2 A^{3 s+2}+(A-1)\right] ; \\
Q_{12 s+7}=\left(A^{3 s+2}-1\right) A^{k-3 s-2}-\left[A^{3 s+2}+(A-1] ; b_{12 s+7}=2 ;\right. \\
P_{12 s+8}=\left(A^{3 s+2}-2\right) A^{k-3 s-2}-(A-1) ; \\
Q_{12 s+8}=4 A^{k-3 s-2} ; b_{12 s+8}=2^{d-1} b A_{3 s+1}-1 ; \\
P_{12 s+9}=\left(A^{3 s+2}-4\right) A^{k-3 s-2}+(A-1) ; \\
Q_{12 s+9}=2\left(A^{3 s+2}-2\right) A^{k-3 s-2}-\left[A^{3(s+1)}-2(A-1)\right] ; b_{12 s+9}=1 ; \\
P_{12 s+10}=A^{k}-\left[A^{3(s+1)}-(A-1)\right] ; \\
Q_{12 s+10}=A^{3(s+1)} ; b_{12 s+10}=2 A^{k-3(s+1)}-2 ; \\
P_{12}{ }^{s}+11=A^{k}-\left[A^{3(s+1)}+(A-1)\right] ; \\
Q_{12 s+11}=2\left(A^{3(s+1)}-2\right) A^{k-3(s+1)}-\left[A^{3(s+1)}+2(A-1)\right] ; \\
b_{12 s+11}=1 ; \\
P_{12 s+12}=\left(A^{3(s+1)}-4\right) A^{k-3(s+1)}-(A-1) ; \\
Q_{12 s+12}=4 A^{k-3(s+1)} ; b_{12 s+12}=2^{d-1} b A_{3 s+2}-1 ; \\
P_{12 s+13}=\left(A^{3(s+1)}-2\right) A^{k-3(s+1)}+(A-1) ; \\
Q_{12 s+13}=\left(A^{3(s+1)}-1\right) A^{k-3(s+1)}-\left[A^{3(s+1)+1}-(A-1)\right] ; \\
b_{12 s+13}=2 ; \\
P_{12 s+14}=A^{k}-\left[2 A^{3(s+1)+1}-(A-1)\right] ; Q_{12 s+14}=4 A^{3(s+1)+1} ; \\
b_{12 s+14}=2^{d-1} b A_{k-3(s+1)-2}-1 \\
s=0, \cdots \\
s=0 \\
\end{array}\right.
$$

Comparison of successive $P_{v}-s$ and $Q_{v}-s$ shows that equality takes place in the following cases:

(14.18) $\left\{\begin{array}{l}P_{12 s+7}=P_{12 s+8} \text { implying } k=2(3 s+2) ; k \equiv 4(\bmod 6) ; \\ s_{0}=\frac{1}{6}(k-4) .\end{array}\right.$ 


$$
\left\{\begin{array}{l}
P_{12 s+13}=P_{12 s+14} \text { implying } k=6(s+1)+1 ; k \equiv 1(\bmod 6) \\
s_{0}=\frac{1}{6}(k-7)
\end{array}\right.
$$

The length of the primitive period in the expansion of $w$ equals, in case (14.18), $m=4+24 \cdot 1 / 6(k-4)+8+2=4 k-2$; in case (14.19) the length of the primitive period equals $m=4+24 \cdot(1 / 6)(k-7)+$ $20+2=4 k-2$. We can now state.

THeOREM 14. Let $A=2^{d} b+1, d \geqq 1, b$ odd,$w^{2}=\left[A^{k}-(A+1)\right]^{2}-$ $4 A$ squarefree; let further $k \equiv 4(\bmod 6), k \geqq 4, s_{0}=(1 / 6)(k-4)$. Then the length of the primitive period in the expansion of $w$ as a periodic continued fraction equals $4 k-2$, and the expansion has the form $k \geqq 10$,

(14.20)

$$
\left\{\begin{array}{l}
w=\left[b_{0}, \overline{b_{1}, b_{2}, \cdots, b_{12 s+3}, b_{12 s+4}, \cdots, b_{12 s+14}, \cdots, b_{12 s_{0}+3}, b_{12 s_{0}+4}},\right. \\
\left.\quad \overline{b_{12 s_{0}+5}, b_{12 s_{0}+6}, 2, b_{12 s_{0}+6}, b_{12 s_{0}+5}, b_{12 s_{0}+4}, b_{12 s_{0}+3}, \cdots, b_{2}, b_{1}, 2 b_{0}}\right] \\
b_{0}=A^{k}-A-2 ; b_{1}=1 ; b_{2}=2^{d-1} b A_{k-2}-2 ; b_{12 s_{0}+3}=b_{12 s_{0}+5}=1 ; \\
b_{12 s_{0}+4}=2 A^{1 / 2(k-2)}-2 ; b_{12 s_{0}+6}=2^{d-1} b A_{1 / 2(k-2)} ; \\
b_{12 s+3}=b_{12 s+5}=\frac{1}{2} b_{12 s+7}=b_{12 s+9}=b_{12 s+11}=\frac{1}{2} b_{12 s+13}=1 ; \\
b_{12 s+4}=2 A^{3 s+1}-2 ; b_{12 s+6}=2^{d-1} b A_{k-3(s+1)}-1 ; \\
b_{12 s+8}=2^{d-1} b A_{3 s+1}-1 ; b_{12 s+10}=2 A^{k-3(s+1)}-2 ; \\
b_{12 s+12}=2^{d-1} b A_{3 s+2}-1 ; b_{12 s+14}=2^{d-1} b A_{k-3 s-5}-1 \\
s=0,1, \cdots, s_{0}-1 ; A^{u+1}-1=(A-1) A_{u} ; u=0,1, \cdots ; \\
A_{0}=1 .
\end{array}\right.
$$

For $k=4$, we obtain the expansion

$$
\left\{\begin{array}{l}
\sqrt{\left[A^{4}-(A+1)\right]^{2}-4 A} \\
=\frac{\left[A^{4}-A-2, \overline{1,2^{d-1} b A_{2}-2,1,2 A-2,1,2^{d-1} b A_{1}, 2}\right.}{\left.2^{d-1} b A_{1}, 1,2 A-2,1,2^{d-1} b A_{2}-2,1,2\left(A^{4}-A-2\right)\right]}
\end{array}\right.
$$

Let further $k \equiv 1(\bmod 6) ; k \geqq 7, s_{0}=1 / 6(k-7)$. Then the length of the period in the expansion of $w$ as a periodic continued fraction equals $4 k-2$, and the expansion has the form

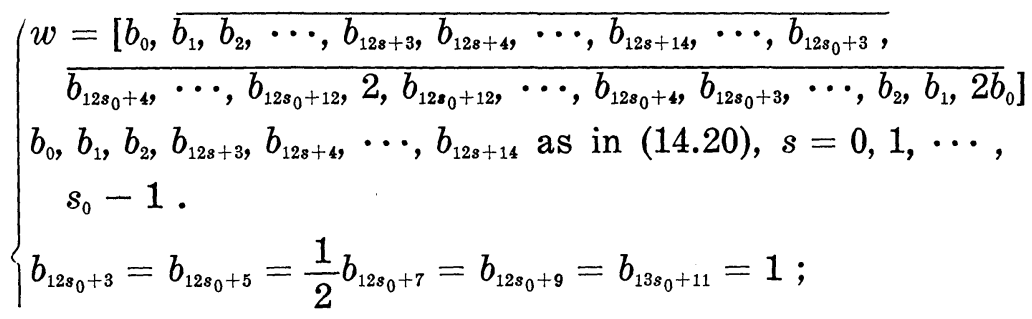




$$
\mid \begin{aligned}
& b_{12 s_{0}+4}=2 A^{1 / 2(k-5)}-2 ; b_{12 s_{0}+6}=2^{d-1} b A_{1 / 2(k+1)}-1 ; \\
& b_{12 s_{0}+8}=2^{d-1} b A_{1 / 2(k-5)}-1 ; b_{12 s_{0}+10}=2 A^{1 / 2(k+1)}-2 ; \\
& b_{12 s_{0}+12}=2^{d-1} b A_{1 / 2(k-3)}-1
\end{aligned}
$$

The reader will have no difficulty to formulate the theorem for the case $k=7$.

\section{REFERENCES}

1. L. Bernstein, The Jacobi-Perron Algorithm, Its Theory and Application, Lecture Notes in Mathematics 207, Springer-Verlag, 1971, 1-160.

2. - Periodicity and Units in Quadratic Number Fields, Submitted.

3. G. Degert, Ueber die Bestimmung der Grundeinheit gewisser real-quadratischer Zahlkoerper, Hamburger Math. Abh., Bd. XXII, (1958), 92-97.

4. Th. Muir, (i) The expression of a quadratic surd as a continued fraction, Glasgow, 1874; (ii) A New Special Class of Determinants, Proc. Ed. 8, 1874.

5. O. Perron, Die Lehre von den Kettenbruechen, Zweite verbeserte Auflage, Chelsea Publ. Co., New York, I-XII + 1-524.

6. Y. Yamamoto, Real quadratic number fields with large fundamental units, Osaka

J. Math., 8, no. 2 (1971) 261-271.

7. H. Zassenhaus, On the units of orders, J. Algebra, 20 (1972), 368-395. 



\section{PACIFIC JOURNAL OF MATHEMATICS}

EDITORS

RICHARD ARENS (Managing Editor)

University of California

Los Angeles, California 90024

R. A. BEAUMONT

University of Washington

Seattle, Washington 98105
J. DugundJI

Department of Mathematics

University of Southern California

Los Angeles, California 90007

D. Gilbarg and J. Milgram

Stanford University

Stanford, California 94305

\section{ASSOCIATE EDITORS}

E. F. BECKENBACH

B. H. NeumanN

F. WOLF

K. YosHIDA

\section{SUPPORTING INSTITUTIONS}

UNIVERSITY OF BRITISH COLUMBIA

UNIVERSITY OF SOUTHERN CALIFORNIA

CALIFORNIA INSTITUTE OF TECHNOLOGY

UNIVERSITY OF CALIFORNIA

STANFORD UNIVERSITY

UNIVERSITY OF TOKYO

MONTANA STATE UNIVERSITY

UNIVERSITY OF UTAH

UNIVERSITY OF NEVADA

WASHINGTON STATE UNIVERSITY

NEW MEXICO STATE UNIVERSITY

UNIVERSITY OF WASHINGTON

OREGON STATE UNIVERSITY

UNIVERSITY OF OREGON

OSAKA UNIVERSITY

AMERICAN MATHEMATICAL SOCIETY
NAVAL WEAPONS CENTER

Printed in Japan by International Academic Printing Co., Ltd., Tokyo, Japan 


\section{Pacific Journal of Mathematics}

\section{Vol. 63, No. $1 \quad$ March, 1976}

Ralph Artino, Gevrey classes and hypoelliptic boundary value problems ....... 1

B. Aupetit, Caractérisation spectrale des algèbres de Banach commutatives .... 23

Leon Bernstein, Fundamental units and cycles in the period of real quadratic

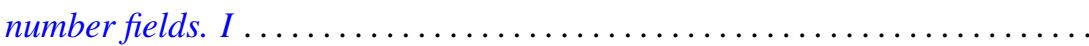

Leon Bernstein, Fundamental units and cycles in the period of real quadratic number fields. II.................................... 63

Robert F. Brown, Fixed points of automorphisms of compact Lie groups ........

Thomas Ashland Chapman, Concordances of noncompact Hilbert cube

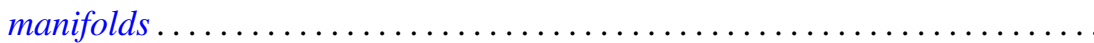

William C. Connett, V and Alan Schwartz, Weak type multipliers for Hankel

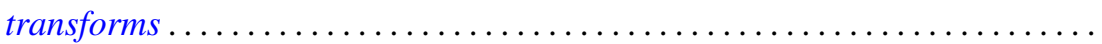

John Wayne Davenport, Multipliers on a Banach algebra with a bounded approximate identity .....................................

Gustave Adam Efroymson, Substitution in Nash functions ................ 137

John Sollion Hsia, Representations by spinor genera ..................

William George Kitto and Daniel Eliot Wulbert, Korovkin approximations in

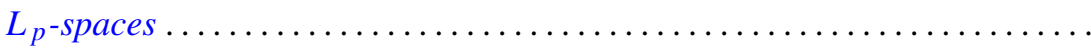

Eric P. Kronstadt, Interpolating sequences for functions satisfying a Lipschitz. condition ...........................................

Gary Douglas Jones and Samuel Murray Rankin, III, Oscillation properties of certain self-adjoint differential equations of the fourth order...

Takaŝi Kusano and Hiroshi Onose, Nonoscillation theorems for differential

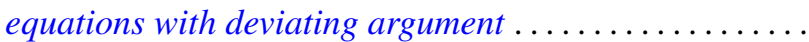

David C. Lantz, Preservation of local properties and chain conditions in commutative group rings. ...

Charles W. Neville, Banach spaces with a restricted Hahn-Banach extension property....

Norman Oler, Spaces of discrete subsets of a locally compact group ...

Robert Olin, Functional relationships between a subnormal operator and its minimal normal extension.

Thomas Thornton Read, Bounds and quantitative comparison theorems for nonoscillatory second order differential equations ...... .

Robert Horace Redfield, Archimedean and basic elements in completely distributive lattice-ordered groups...

Jeffery William Sanders, Weighted Sidon sets

Aaron R. Todd, Continuous linear images of pseudo-complete linear topological spaces.

J. Jerry Uhl, Jr., Norm attaining operators on $L^{1}[0,1]$ and the Radon-Nikodým property.

William Jennings Wickless, Abelian groups in which every endomorphism is a left multiplication. 